\title{
Numerical Solutions of Fractional Integrodifferential Equations of Bratu Type by Using CAS Wavelets
}

\author{
Mingxu Yi, Kangwen Sun, Jun Huang, and Lifeng Wang \\ School of Aeronautical Science and Technology, Beihang University, Beijing 100191, China \\ Correspondence should be addressed to Kangwen Sun; sunkw1900@163.com
}

Received 28 September 2013; Accepted 31 October 2013

Academic Editor: Ning Hu

Copyright (C) 2013 Mingxu Yi et al. This is an open access article distributed under the Creative Commons Attribution License, which permits unrestricted use, distribution, and reproduction in any medium, provided the original work is properly cited.

\begin{abstract}
A numerical method based on the CAS wavelets is presented for the fractional integrodifferential equations of Bratu type. The CAS wavelets operational matrix of fractional order integration is derived. A truncated CAS wavelets series together with this operational matrix is utilized to reduce the fractional integrodifferential equations to a system of algebraic equations. The solution of this system gives the approximation solution for the truncated limited $2^{k}(2 M+1)$. The convergence and error estimation of CAS wavelets are also given. Two examples are included to demonstrate the validity and applicability of the approach.
\end{abstract}

\section{Introduction}

A lot of scientific and engineering problems involving fractional phenomenon are already very large and still growing. One of the main advantages of the fractional phenomenon is that the fractional derivatives and fractional integrals provide an excellent approach to the different kinds of physical fields, such as dispersive transports in amorphous semiconductors, tracer transfer in underground water, and seepage in soil or rocks [1-4]. In recent years, more and more researchers are finding that a variety of dynamical problems exhibit fractional order behavior. This indicates that variable order calculus is an effective mathematical framework to describe the complex dynamical problems [5-7]. Many of the numerical methods using various fractional derivative operators and integral operators for solving fractional differential equations have been proposed. Podlubny [8] used the Laplace transform method to solve the fractional partial differential equations with constant coefficients. Odibat and Momani [9] applied generalized differential transform method to solve the numerical solution of linear partial differential equations of fractional order. Zhang [10] discussed a practical implicit method to solve a class of initial boundary value spacetime fractional convection-diffusion equations with variable coefficients. Zhuang et al. [11] proposed explicit and implicit
Euler method for the variable order fractional advectiondiffusion equation.

Bratu's problem is also discussed in all kinds of applications, such as chemical reaction theory, the fuel ignition model of the thermal combustion theory, and nanotechnology [12-15]. Both mathematicians and physicists have devoted a lot of effort to Bratu's problem. In [16], Syam and Hamdan presented the Laplace Adomian decomposition method for solving Bratu's problem. Wazwaz [17] proposed the Adomian decomposition method for solving Bratu's problem. Aksoy and Pakdemirli [18] had solved Bratu-type equation of new perturbation iteration solutions.

In this paper, we consider the following fractional integrodifferential equations of Bratu type by means of CAS wavelets:

$$
\begin{array}{r}
D^{\alpha} u(x)+\lambda \int_{0}^{x} k(x, t) e^{u(t)} d t+g(x)=0, \\
n-1<\alpha \leq n, 0 \leq x, t \leq 1,
\end{array}
$$

with initial condition

$$
u^{(j)}(0)=b_{j}, \quad j=0,1, \ldots, n-1 .
$$




\section{Definitions and Properties of Fractional Operator}

Here we just recall the most typical definitions which are easy to use in physics. The Caputo fractional differential operator $D^{\alpha}$ of order $\alpha>0$ is defined as [8]

$$
D^{\alpha} f(t)=\frac{1}{\Gamma(n-\alpha)} \int_{0}^{t}(t-\tau)^{n-\alpha-1} f^{(n)}(\tau) d \tau .
$$

The Riemann-Liouville fractional integration of order $\alpha>0$ is defined as

$$
I^{\alpha} f(t)=\frac{1}{\Gamma(\alpha)} \int_{0}^{t}(t-\tau)^{\alpha-1} f(\tau) d \tau, \quad n-1<\alpha \leq n
$$

where $n$ is a positive integer.

It has the following two basic properties for $n-1<\alpha \leq n$ :

$$
\begin{gathered}
D^{\alpha} I^{\alpha} f(t)=f(t), \\
I^{\alpha} D^{\alpha} f(t)=f(t)-\sum_{k=0}^{n-1} f^{(k)}\left(0^{+}\right) \frac{t^{k}}{k !}, \quad t>0 .
\end{gathered}
$$

\section{CAS Wavelets and Some of Their Properties}

3.1. CAS Wavelets. The CAS wavelets $\psi_{n m}(t)=\psi(k, n, m, t)$ have four arguments, $n=1,2, \ldots, 2^{k}$, where $k$ is any nonnegative integer, $m$ is any integer, and $t$ is the normalized time. The orthonormal CAS wavelets are defined on the interval $[0,1)$ by $[19]$

$$
\psi_{n m}(t)= \begin{cases}2^{k / 2} \mathrm{CAS}_{m}\left(2^{k} t-n+1\right), & \frac{n-1}{2^{k}} \leq t<\frac{n}{2^{k}} \\ 0, & \text { otherwise, }\end{cases}
$$

where $\mathrm{CAS}_{m}(t)=\cos (2 m \pi t)+\sin (2 m \pi t)$.

3.2. Function Approximation. A function $u(t) \in L^{2}[0,1)$ may be expanded as

$$
u(t)=\sum_{n=1}^{\infty} \sum_{m \in Z} c_{n m} \psi_{n m}(t)
$$

where $c_{n m}=\left\langle u(t), \psi_{n m}(t)\right\rangle$, in which \langle\rangle denotes the inner product. The series (7) is truncated as

$$
u(t) \approx u_{k, M}(t)=\sum_{n=1}^{2^{k}} \sum_{m=-M}^{M} c_{n m} \psi_{n m}=C^{T} \Psi(t),
$$

where $C$ and $\Psi(t)$ are two vectors given by

$$
\begin{aligned}
C= & {\left[c_{1(-M)}, c_{1(-M+1)}, \ldots c_{1(M)}, c_{2(-M)}, \ldots,\right.} \\
& \left.c_{2(M)}, \ldots, c_{2^{k}(-M)}, \ldots c_{2^{k}(M)}\right]^{T} \\
= & {\left[c_{1}, c_{2}, \ldots, c_{2^{k}(2 M+1)}\right]^{T}, } \\
\Psi(t)= & {\left[\psi_{1(-M)}, \psi_{1(-M+1)}, \ldots, \psi_{1(M)}, \psi_{2(-M)}, \ldots,\right.} \\
& \left.\psi_{2(M)}, \psi_{2^{k}(-M)}, \ldots, \psi_{2^{k}(M)}\right]^{T} \\
= & {\left[\psi_{1}, \psi_{2}, \ldots, \psi_{2^{k}(2 M+1)}\right]^{T} . }
\end{aligned}
$$

3.3. Convergence of the CAS Wavelet Bases. In this section, we indicate that the CAS wavelets expansion of a function $u(t)$, with bounded second derivative, converges uniformly to $u(t)$.

Lemma 1. If the CAS wavelet expansion of a continuous function $u(t)$ converges uniformly, then the CAS wavelet expansion converges to the function $u(t)$.

Proof. Let

$$
v(t)=\sum_{n=1}^{\infty} \sum_{m \in Z} c_{n m} \psi_{n m}(t),
$$

where $c_{n m}=\left\langle u(t), \psi_{n m}(t)\right\rangle$. Multiplying both sides of (11) by $\psi_{p q}(t), p$, and $q$ are fixed and then integrating termwise, justified by uniformly convergence, on $[0,1]$, we have

$$
\begin{aligned}
\int_{0}^{1} v(t) & \psi_{p q}(t) d t \\
& =\int_{0}^{1} \sum_{n=1}^{\infty} \sum_{m \in Z} c_{n m} \psi_{n m}(t) \psi_{p q}(t) d t \\
& =\sum_{n=1}^{\infty} \sum_{m \in Z} c_{n m} \int_{0}^{1} \psi_{n m}(t) \psi_{p q}(t) d t=c_{p q} .
\end{aligned}
$$

Thus $\left\langle v(t), \psi_{n m}(t)\right\rangle=c_{n m}$ for $n, m \in Z$. Consequently $u, v$ have same Fourier expansions with the CAS wavelets basis and therefore $u(t)=v(t)$.

Theorem 2. A function $u(t) \in L^{2}[0,1]$, with bounded second derivative, say $\left|u^{\prime \prime}(t)\right| \leq \gamma$, can be expanded as an infinite sum of the CAS wavelets and the series converges uniformly to $u(t)$; that is $u(t)=\sum_{n=1}^{\infty} \sum_{m \in Z} c_{n m} \psi_{n m}(t)$. Furthermore, we have

$$
\left|u_{k, M}(t)-u(t)\right| \leq \frac{\gamma}{\pi^{2}} \sum_{n=2^{k}}^{\infty} \sum_{m=M+1}^{\infty} \frac{1}{n^{5 / 2} m^{2}}, \quad t \in[0,1] .
$$

Proof. From (6), we obtain

$$
\begin{aligned}
c_{n m} & =\int_{0}^{1} u(t) \psi_{n m}(t) d t \\
& =\int_{(n-1) / 2^{k}}^{n / 2^{k}} 2^{k / 2} u(t) \mathrm{CAS}_{m}\left(2^{k} t-n+1\right) d t .
\end{aligned}
$$


Substituting $2^{k} t-n+1=x$ in (13) yields

$$
\begin{aligned}
c_{n m}= & \frac{1}{2^{k / 2}} \int_{0}^{1} u\left(\frac{x+n-1}{2^{k}}\right) \operatorname{CAS}_{m}(x) d x \\
= & \frac{1}{2^{k / 2}} \int_{0}^{1} u\left(\frac{x+n-1}{2^{k}}\right) d\left(\frac{\sin (2 m \pi x)-\cos (2 m \pi x)}{2 m \pi}\right) \\
= & \left.\frac{1}{2^{k / 2}} u\left(\frac{x+n-1}{2^{k}}\right) \frac{\sin (2 m \pi x)-\cos (2 m \pi x)}{2 m \pi}\right|_{0} ^{1} \\
& -\frac{1}{2^{3 k / 2}(2 m \pi)} \int_{0}^{1} u^{\prime}\left(\frac{x+n-1}{2^{k}}\right) \\
& \times(\sin (2 m \pi x)-\cos (2 m \pi x)) d x \\
= & \frac{1}{2^{3 k / 2}(2 m \pi)} \int_{0}^{1} u^{\prime}\left(\frac{x+n-1}{2^{k}}\right) d\left(\frac{\operatorname{CAS}_{m}(x)}{2 m \pi}\right) \\
= & \left.\frac{1}{2^{3 k / 2}(2 m \pi)^{2}} u^{\prime}\left(\frac{x+n-1}{2^{k}}\right) \operatorname{CAS}_{m}(x)\right|_{0} ^{1} \\
& -\frac{1}{2^{5 k / 2}(2 m \pi)^{2}} \int_{0}^{1} u^{\prime \prime}\left(\frac{x+n-1}{2^{k}}\right) \operatorname{CAS}_{m}(x) d x .
\end{aligned}
$$

Thus, we get

$$
\begin{aligned}
\left|c_{n m}\right|^{2}= & \left|\frac{1}{2^{5 k / 2}(2 m \pi)^{2}} \int_{0}^{1} u^{\prime \prime}\left(\frac{x+n-1}{2^{k}}\right) \operatorname{CAS}_{m}(x) d x\right|^{2} \\
\leq & \left(\frac{1}{2^{5 k / 2}(2 m \pi)^{2}}\right)^{2} \int_{0}^{1}\left|u^{\prime \prime}\left(\frac{x+n-1}{2^{k}}\right)\right|^{2} d x \\
& \times \int_{0}^{1}\left|\mathrm{CAS}_{m}(x)\right|^{2} d x \\
\leq & \left(\frac{\gamma}{2^{5 k / 2}(2 m \pi)^{2}}\right)^{2} \int_{0}^{1}\left|\operatorname{CAS}_{m}(x)\right|^{2} d x
\end{aligned}
$$

from orthonormality of CAS wavelets, we know that $\int_{0}^{1}\left|\mathrm{CAS}_{m}(x)\right|^{2} d x=1$, since $n \leq 2^{k}$, we have $\left|c_{n m}\right| \leq$ $\gamma /\left(4 \pi^{2} n^{5 / 2} m^{2}\right)$. Hence the series $\sum_{n=1}^{\infty} \sum_{m \in Z} c_{n m}$ is absolutely convergent. On the other hand, we can obtain

$$
\begin{aligned}
& \left|\sum_{n=1}^{\infty} \sum_{m \in Z} c_{n m} \psi_{n m}(t)\right| \\
& \quad \leq \sum_{n=1}^{\infty} \sum_{m \in Z}\left|c_{n m}\right|\left|\psi_{n m}(t)\right| \leq 2 \sum_{n=1}^{\infty} \sum_{m \in Z}\left|c_{n m}\right|<\infty .
\end{aligned}
$$

Using Lemma 1 , the series $\sum_{n=1}^{\infty} \sum_{m \in Z} c_{n m} \psi_{n m}(t)$ converges to $u(t)$.
Moreover, we conclude that

$$
\begin{aligned}
\left|u_{k, M}(t)-u(t)\right| & \leq\left|2 \sum_{n=2^{k}+1}^{\infty} \sum_{m=M+1}^{\infty} c_{n m} \psi_{n m}(t)\right| \\
& \leq 4 \sum_{n=2^{k}+1}^{\infty} \sum_{m=M+1}^{\infty}\left|c_{n m}\right| \\
& \leq \frac{\gamma}{\pi^{2}} \sum_{n=2^{k}}^{\infty} \sum_{m=M+1}^{\infty} \frac{1}{n^{5 / 2} m^{2}} .
\end{aligned}
$$

This completes the proof.

3.4. Operational Matrix of the Fractional Integration. In this part, we may simply introduce the operational matrix of fractional integration of CAS wavelets; more detailed introduction can be found in [19].

Take the points $t_{i}=i / 2^{k}(2 M+1), i=0,1,2, \ldots, 2^{k}(2 M+$ 1) -1 , then we define

$$
\Phi=\left[\Psi\left(t_{0}\right) \Psi\left(t_{1}\right) \cdots \Psi\left(t_{2^{k}(2 M+1)-1}\right)\right] .
$$

If $J^{\alpha}$ is fractional integration operator of CAS wavelets, we can get

$$
\left(J^{\alpha} \Psi\right)(t)=P^{\alpha} \Psi(t),
$$

where $P^{\alpha}$ is called operational matrix of fractional integration of CAS wavelets.

Apart from the CAS wavelets, we consider another basis set of block pulse functions. The set of these functions, over the interval $[0,1)$, is defined as

$$
b_{i}(t)= \begin{cases}1, & \bar{l} \leq t<\frac{i+1}{l}, i=0,1,2, \ldots, l-1 \\ 0, & \text { otherwise, }\end{cases}
$$

with a positive integer value for $l$, we suppose $l=2^{k}(2 M+1)$ in this paper.

Let $B_{l}(t)=\left[b_{0}(t), b_{1}(t), \ldots, b_{l-1}(t)\right]^{T}$, then the block pulse functions operational matrix of fractional integration $F^{\alpha}$ is given by [20]

$$
\left(J^{\alpha} B_{l}\right)(t)=F^{\alpha} B_{l}(t),
$$

where

$$
\begin{gathered}
F^{\alpha}=\frac{1}{l^{\alpha}} \frac{1}{\Gamma(\alpha+2)}\left[\begin{array}{ccccc}
1 & \xi_{1} & \xi_{2} & \cdots & \xi_{l-1} \\
0 & 1 & \xi_{1} & \cdots & \xi_{l-2} \\
0 & 0 & 1 & \cdots & \xi_{l-3} \\
\vdots & \vdots & \vdots & \vdots & \vdots \\
0 & 0 & 0 & \cdots & 1
\end{array}\right], \\
\xi_{k}=(k+1)^{\alpha+1}-2 k^{\alpha+1}+(k-1)^{\alpha+1} .
\end{gathered}
$$

There is a relation between the block pulse functions and CAS wavelets:

$$
\Psi(t)=\Phi B_{l}(t) .
$$

Therefore we can derive $P^{\alpha}$ easily by using (19), (21), and (23):

$$
P^{\alpha}=\Phi F^{\alpha} \Phi^{-1}
$$




\section{Numerical Solution of (1)-(2)}

Consider the nonlinear fractional integrodifferential equations of Bratu type

$$
\begin{aligned}
& D^{\alpha} u(x)+\lambda \int_{0}^{x} k(x, t) e^{u(t)} d t+g(x)=0, \\
& u^{(j)}(0)=b_{j}, \quad 0 \leq x, t \leq 1, n-1<\alpha \leq n,
\end{aligned}
$$

where $\lambda$ is arbitrary parameter, $k(x, t) \in L^{2}([0,1] \times[0,1])$, and $g(x)$ is a known function.

Let $D^{\alpha} u(x) \approx C^{T} \Psi(x), g(x) \approx G^{T} \Psi(x), k(x, t) \approx$ $\Psi^{T}(x) K \Psi(t)$, and $e^{u(t)} \approx \sum_{i=0}^{l}[u(t)]^{i} / i !$, then

$$
\begin{aligned}
u(x) & \approx C^{T} P^{\alpha} \Psi(x)+\sum_{k=0}^{n-1} u^{(k)}(0) \frac{x^{k}}{k !} \\
& =C^{T} P^{\alpha} \Psi(x)+Q \Psi(x)=\left(C^{T} P^{\alpha}+Q\right) \Psi(x),
\end{aligned}
$$

where coefficient $Q$ is known and can be obtained by using the initial conditions.

Substituting (23) into (26), we obtain

$$
u(x) \approx\left(C^{T} P^{\alpha}+Q\right) \Psi(x)=\left(C^{T} P^{\alpha}+Q\right) \Phi B_{l}(x) .
$$

Define $\left(C^{T} P^{\alpha}+Q\right) \Phi=\left[a_{1}, a_{2}, \ldots, a_{m}\right]=A$, then $u(x) \approx$ $A B_{l}(x)$.

Applying the properties of BPFs, we have

$$
[u(x)]^{i} \approx\left[a_{1}^{i}, a_{2}^{i}, \ldots, a_{m}^{i}\right] B_{l}(x)=A_{i} B_{l}(x) .
$$

By substituting the above expanded forms into (1), we get

$$
\begin{aligned}
D^{\alpha} & u(x)+\lambda \int_{0}^{x} k(x, t) e^{u(t)} d t+g(x) \\
\approx & C^{T} \Psi(x)+\lambda \int_{0}^{x} \Psi^{T}(x) K \Psi(t) \sum_{j=0}^{l} \frac{[u(t)]^{j}}{j !} d t+G^{T} \Psi(x) \\
\approx & C^{T} \Psi(x)+\lambda \Psi^{T}(x) K \\
& \times \int_{0}^{x} \Phi B_{l}(t) \sum_{j=0}^{l} \frac{B_{l}^{T}(t) A_{j}^{T}}{j !} d t+G^{T} \Psi(x) \\
\approx & C^{T} \Psi(x)+\lambda \Psi^{T}(x) K \Phi \sum_{j=0}^{l} \frac{1}{j !} \int_{0}^{x} \widetilde{A}_{j}^{T} B_{l}(t) d t+G^{T} \Psi(x) \\
\approx & C^{T} \Psi(x)+\lambda \Psi^{T}(x) K \Phi \sum_{j=0}^{l} \frac{1}{j !} \widetilde{A}_{j}^{T} F^{1} B_{l}(x)+G^{T} \Psi(x)=0,
\end{aligned}
$$

where $\widetilde{A}_{j}^{T}$ is the product operational matrix of $A_{j}^{T}$.

Putting the collocation points $\left\{x_{i}\right\}_{i=0}^{2^{k}(2 M+1)-1}$ into (29), (29) will be

$$
C^{T} \Psi\left(x_{i}\right)+\lambda \Psi^{T}\left(x_{i}\right) K \Phi \sum_{j=0}^{l} \frac{1}{j !} \widetilde{A}_{j}^{T} F^{1} B_{l}\left(x_{i}\right)+G^{T} \Psi\left(x_{i}\right)=0 .
$$

Solving the nonlinear algebraic equations (30) by using Newton iteration method, we obtain the vector $C^{T}$, and then we get the approximate solution $u(x)=\left(C^{T} P^{\alpha}+Q\right) \Psi(x)$.

\section{Existence of Uniqueness}

Theorem 3 (uniqueness theorem). Equation (1) has a unique solution whenever $0<\beta<1$, where

$$
\begin{gathered}
\beta=\frac{\lambda M L}{\Gamma(\alpha) \alpha(\alpha+1)}, \\
M=\sup _{0 \leq x, t \leq 1}|k(x, t)|<\infty .
\end{gathered}
$$

Proof. Suppose $t \in[0,1]$, then $u(t)$ is bounded. Therefore the nonlinear term $e^{u(t)}$ in (1) is Lipschitz continuous with $\mid e^{u}-$ $e^{u_{*}}|\leq L| u-u_{*} \mid, L>0$.

Let $u$ and $u^{*}$ be two different solutions of (1), then we can get

$$
\begin{gathered}
D^{\alpha} u(x)=-\lambda \int_{0}^{x} k(x, t) e^{u(t)} d t-g(x), \\
D^{\alpha} u_{*}(x)=-\lambda \int_{0}^{x} k(x, t) e^{u_{*}(t)} d t-g(x) .
\end{gathered}
$$

Using Riemann-Liouville fractional integration, we have

$$
\begin{aligned}
I^{\alpha} D^{\alpha} u(x)= & -\frac{1}{\Gamma(\alpha)} \int_{0}^{x}(x-\xi)^{\alpha-1} g(\xi) d \xi \\
& -\frac{\lambda}{\Gamma(\alpha)} \int_{0}^{x} \int_{0}^{\xi}(x-\xi)^{\alpha-1} k(\xi, t) e^{u(t)} d t d \xi, \\
I^{\alpha} D^{\alpha} u_{*}(x)= & -\frac{1}{\Gamma(\alpha)} \int_{0}^{x}(x-\xi)^{\alpha-1} g(\xi) d \xi \\
& -\frac{\lambda}{\Gamma(\alpha)} \int_{0}^{x} \int_{0}^{\xi}(x-\xi)^{\alpha-1} k(\xi, t) e^{u_{*}(t)} d t d \xi .
\end{aligned}
$$

Because $I^{\alpha} D^{\alpha} x(t)=x(t)-\sum_{k=0}^{n-1}\left(t^{k} / k !\right) x^{(k)}\left(0^{+}\right)$, so (33) can transform as

$$
\begin{aligned}
u(x)= & \sum_{j=0}^{n-1} \frac{x^{j}}{j !} u^{(j)}(0) \\
= & -\frac{1}{\Gamma(\alpha)} \int_{0}^{x}(x-\xi)^{\alpha-1} g(\xi) d \xi \\
& -\frac{\lambda}{\Gamma(\alpha)} \int_{0}^{x} \int_{0}^{\xi}(x-\xi)^{\alpha-1} k(\xi, t) e^{u(t)} d t d \xi, \\
u_{*}(x)- & \sum_{j=0}^{n-1} \frac{x^{j}}{j !} u_{*}^{(j)}(0) \\
= & -\frac{1}{\Gamma(\alpha)} \int_{0}^{x}(x-\xi)^{\alpha-1} g(\xi) d \xi \\
& -\frac{\lambda}{\Gamma(\alpha)} \int_{0}^{x} \int_{0}^{\xi}(x-\xi)^{\alpha-1} k(\xi, t) e^{u_{*}(t)} d t d \xi .
\end{aligned}
$$


TABLE 1: Absolute errors for different values of $k, M$.

\begin{tabular}{lccc}
\hline$t$ & $k=1, M=1$ & $k=2, M=1$ & $k=3, M=1$ \\
\hline 0 & 0.05345058698637 & 0.02590432861120 & 0.00591634971861 \\
$1 / 6$ & 0.06527407677339 & 0.03850276248440 & 0.00861046012104 \\
$2 / 6$ & 0.05142870790491 & 0.01273940964104 & 0.00925569874104 \\
$3 / 6$ & 0.02376570358066 & 0.00782417705008 & 0.00174295063041 \\
$4 / 6$ & 0.03786855145819 & 0.00811001255098 & 0.00314673017636 \\
$5 / 6$ & 0.08725888105360 & 0.04440645099374 & 0.03718748499037 \\
\hline
\end{tabular}

Then we have

$$
\begin{aligned}
& \left|u-u_{*}\right| \\
& =\mid-\frac{\lambda}{\Gamma(\alpha)} \int_{0}^{x} \int_{0}^{\xi}(x-\xi)^{\alpha-1} k(\xi, t) e^{u(t)} d t d \xi \\
& +\frac{\lambda}{\Gamma(\alpha)} \int_{0}^{x} \int_{0}^{\xi}(x-\xi)^{\alpha-1} k(\xi, t) e^{u_{*}(t)} d t d \xi \\
& =\left|-\frac{\lambda}{\Gamma(\alpha)} \int_{0}^{x} \int_{0}^{\xi}(x-\xi)^{\alpha-1} k(\xi, t)\left[e^{u(t)}-e^{u_{*}(t)}\right] d t d \xi\right| \\
& \leq \frac{\lambda}{\Gamma(\alpha)} \int_{0}^{x} \int_{0}^{\xi}(x-\xi)^{\alpha-1} k(\xi, t)\left|e^{u(t)}-e^{u_{*}(t)}\right| d t d \xi \\
& \leq \frac{\lambda M L}{\Gamma(\alpha)} \int_{0}^{x} \int_{0}^{\xi}(x-\xi)^{\alpha-1}\left|u-u_{*}\right| d t d \xi \\
& =\frac{\lambda M L}{\Gamma(\alpha)} \int_{0}^{x}(x-\xi)^{\alpha-1}\left|u-u_{*}\right| \xi d \xi \\
& =\frac{\lambda M L}{\Gamma(\alpha)}\left|u-u_{*}\right| \int_{0}^{x} \xi(x-\xi)^{\alpha-1} d \xi \\
& =\frac{\lambda M L}{\Gamma(\alpha)}\left|u-u_{*}\right|\left(\frac{x^{\alpha+1}}{\alpha}-\frac{x^{\alpha+1}}{\alpha+1}\right) \\
& =\frac{\lambda M L}{\Gamma(\alpha)}\left|u-u_{*}\right| x^{\alpha+1} \frac{1}{\alpha(\alpha+1)} \\
& \leq \frac{\lambda M L}{\Gamma(\alpha) \alpha(\alpha+1)}\left|u-u_{*}\right| \text {. }
\end{aligned}
$$

Therefore $\left|u-u_{*}\right|(1-(\lambda M L / \Gamma(\alpha) \alpha(\alpha+1))) \leq 0$.

This implies that $\left|u-u_{*}\right|(1-\beta) \leq 0$, where $\beta=$ $\lambda M L / \Gamma(\alpha) \alpha(\alpha+1)$.

As $0<\beta<1,\left|u-u_{*}\right|=0$; implying $u=u_{*}$, we can prove (1) has the uniqueness solution.

\section{Numerical Examples}

To show the efficiency and the accuracy of the proposed method, we consider the following two examples.

Example 1. Consider the following equation:

$$
D^{0.5} u(x)+\int_{0}^{x} x\left(t-\frac{1}{2}\right) e^{u(t)} d t+g(x)=0,
$$

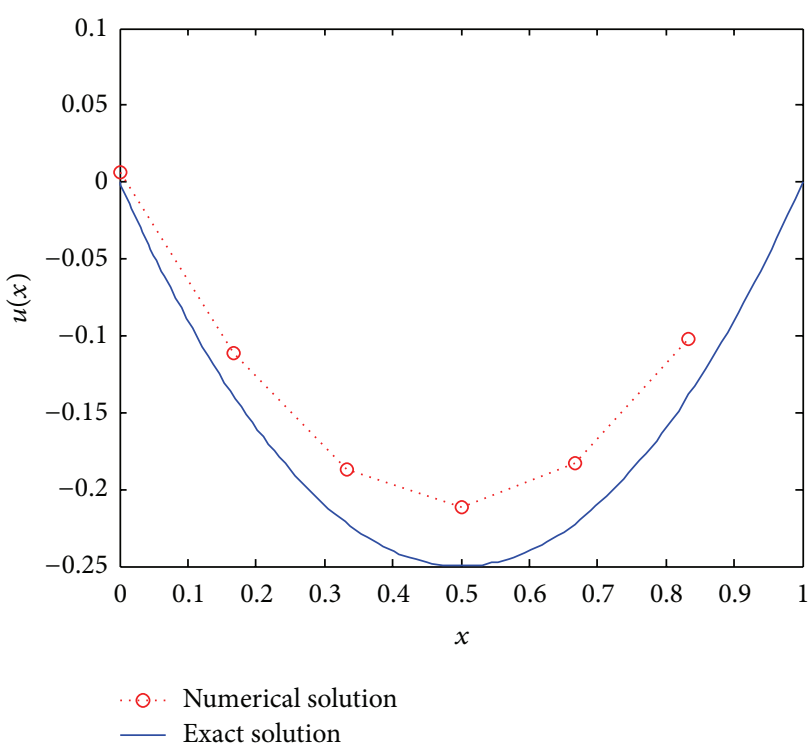

FIGURE 1: The comparison between numerical and exact solution for $k=1, M=1$.

with this condition $u(0)=0$ and $g(x)=-(\Gamma(3) / \Gamma(2.5)) x^{1.5}+$ $(\Gamma(2) / \Gamma(1.5)) x^{0.5}-(x / 2)\left(e^{x^{2}-x}-1\right)$. The exact solution of this problem is $u(x)=x^{2}-x$. Table 1 shows the absolute errors of the approximate solutions and the exact solution. The comparison between the numerical solutions and the exact solution for different $k$ is shown in Figures 1, 2, and 3.

Taking a closer look at Table 1 and Figures $1-3$, with $k$ increasing, we find that the approximate solutions converge to the exact solution.

Example 2. Consider this equation

$$
D^{\alpha} u(x)+3 \int_{0}^{x}(x-t) e^{u(t)} d t+g(x)=0, \quad 0<x, t<1,
$$

such that the initial conditions $u(0)=0, g(x)=-(1 / 2) x^{3}-$ $(1 / x)$. The exact solution of the problem for $\alpha=1$ is given by $u(x)=\ln x$. The comparison of numerical results for $\alpha=$ $0.5, \alpha=0.75$, and $\alpha=1$ and the exact solution for $\alpha=1$ are shown in Figure 4. From Figure 4, we can see clearly that the numerical solutions are in very good agreement with the exact solution when $\alpha=1$. 


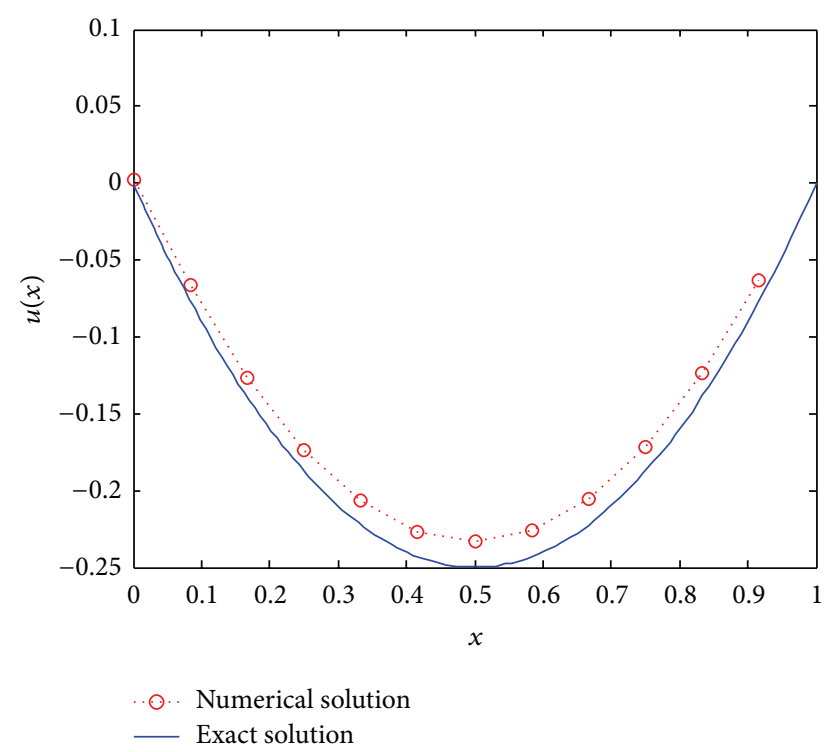

FIGURE 2: The comparison between numerical and exact solution for $k=2, M=1$.

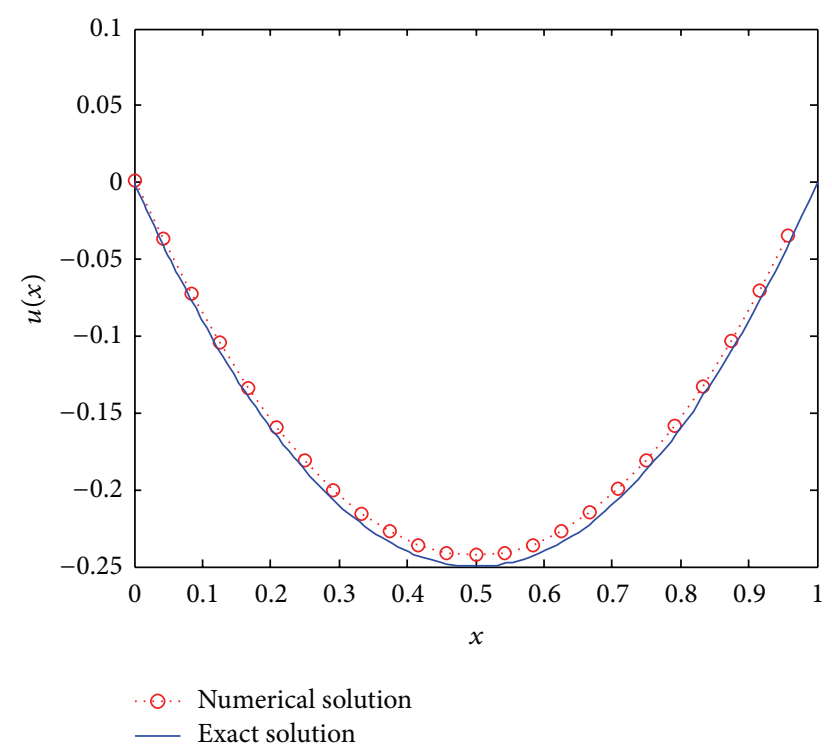

FIgURE 3: The comparison between numerical and exact solution for $k=3, M=1$.

It is evident from Figure 4 that, as $\alpha$ close to 1 , the numerical solution of the CAS wavelets converges to the exact solution; that is, the solution of fractional integrodifferential equation approaches to the solution of integer order integrodifferential equation.

\section{Conclusion}

In this paper, a numerical method is presented by numerical solutions of fractional integrodifferential equations of Bratu type. Taking full advantage of the definition of Caputo type fractional derivative and the properties of CAS wavelet, we

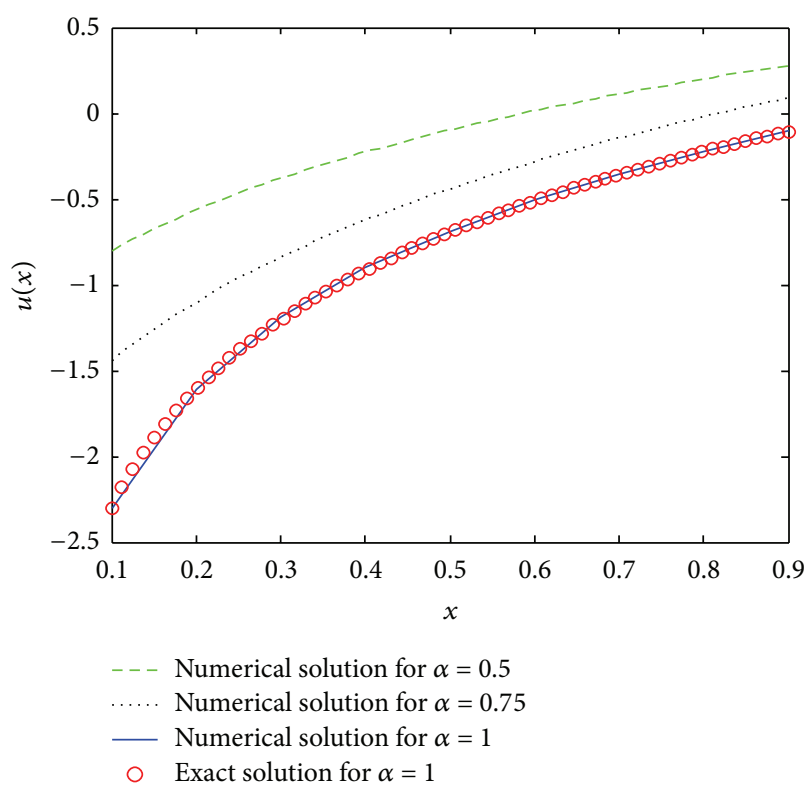

Figure 4: Numerical solution and exact solution of $\alpha=1$.

transform the initial problem into a nonlinear algebraic system equation. By solving the nonlinear system, numerical solutions are obtained. The convergence analysis of CAS wavelets and the uniqueness theorem of this equation are proposed. The numerical results show that the approximation is in very good coincidence with the exact solution.

\section{Acknowledgment}

This paper was supported by the International Science \& Technology Cooperation Program of China (no. 2012DFG61930).

\section{References}

[1] V. V. Anh, J. M. Angulo, and M. D. Ruiz-Medina, "Diffusion on multifractals," Nonlinear Analysis, Theory, Methods and Applications, vol. 63, no. 5-7, pp. e2043-e2056, 2005.

[2] W. Chen, "A speculative study of $2 / 3$-order fractional Laplacian modeling of turbulence: some thoughts and conjectures," Chaos, vol. 16, no. 2, Article ID 023126, 2006.

[3] M. M. Meerschaert and C. Tadjeran, "Finite difference approximations for fractional advection-dispersion flow equations," Journal of Computational and Applied Mathematics, vol. 172, no. 1, pp. 65-77, 2004.

[4] H. Sun, W. Chen, H. Sheng, and Y. Chen, "On mean square displacement behaviors of anomalous diffusions with variable and random orders," Physics Letters A, vol. 374, no. 7, pp. 906910, 2010.

[5] V. V. Anh and N. N. Leonenko, "Spectral analysis of fractional kinetic equations with random data," Journal of Statistical Physics, vol. 104, no. 5-6, pp. 1349-1387, 2001.

[6] T. Blaszczyk, M. Ciesielski, M. Klimek, and J. Leszczynski, "Numerical solution of fractional oscillator equation," Applied Mathematics and Computation, vol. 218, no. 6, pp. 2480-2488, 2011. 
[7] C.-M. Chen, F. Liu, V. Anh, and I. Turner, "Numerical schemes with high spatial accuracy for a variable-order anomalous subdiffusion equation," SIAM Journal on Scientific Computing, vol. 32, no. 4, pp. 1740-1760, 2010.

[8] I. Podlubny, Fractional Differential Equations, Mathematics in Science and Engineering, Academic Press, San Diego, Calif, USA, 1999.

[9] Z. Odibat and S. Momani, "A generalized differential transform method for linear partial differential equations of fractional order," Applied Mathematics Letters, vol. 21, no. 2, pp. 194-199, 2008.

[10] Y. Zhang, "A finite difference method for fractional partial differential equation," Applied Mathematics and Computation, vol. 215, no. 2, pp. 524-529, 2009.

[11] P. Zhuang, F. Liu, V. Anh, and I. Turner, "Numerical methods for the variable-order fractional advection-diffusion equation with a nonlinear source term," SIAM Journal on Numerical Analysis, vol. 47, no. 3, pp. 1760-1781, 2009.

[12] C. K. Chui, Wavelets: A Mathematical Tool for Signal Processing, SIAM Monographs on Mathematical Modeling and Computation, SIAM, Philadelphia, Pa, USA, 1997.

[13] H. T. Davis, Introduction to Nonlinear Differential and Integral Equations, Dover, New York, NY, USA, 1962.

[14] D. A. Frank-Kamenetski, Diffusion and Heat Exchange in Chemical Kinetics, Princeton University Press, Princeton, NJ, USA, 1955.

[15] I. H. A. H. Hassan and V. S. Ertürk, "Applying differential transformation method to the one-dimensional planar Bratu problem," International Journal of Contemporary Mathematical Sciences, vol. 2, no. 29-32, pp. 1493-1504, 2007.

[16] M. I. Syam and A. Hamdan, "An efficient method for solving Bratu equations," Applied Mathematics and Computation, vol. 176, no. 2, pp. 704-713, 2006.

[17] A.-M. Wazwaz, "Adomian decomposition method for a reliable treatment of the Bratu-type equations," Applied Mathematics and Computation, vol. 166, no. 3, pp. 652-663, 2005.

[18] Y. Aksoy and M. Pakdemirli, "New perturbation-iteration solutions for Bratu-type equations," Computers \& Mathematics with Applications, vol. 59, no. 8, pp. 2802-2808, 2010.

[19] H. Saeedi and M. M. Moghadam, "Numerical solution of nonlinear Volterra integro-differential equations of arbitrary order by CAS wavelets," Communications in Nonlinear Science and Numerical Simulation, vol. 16, no. 3, pp. 1216-1226, 2011.

[20] Y. Li and N. Sun, "Numerical solution of fractional differential equations using the generalized block pulse operational matrix," Computers \& Mathematics with Applications, vol. 62, no. 3, pp. 1046-1054, 2011. 


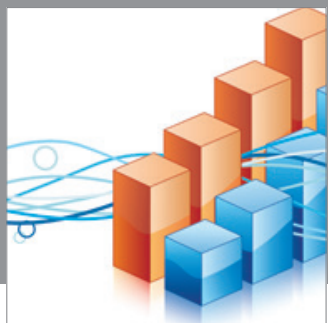

Advances in

Operations Research

mansans

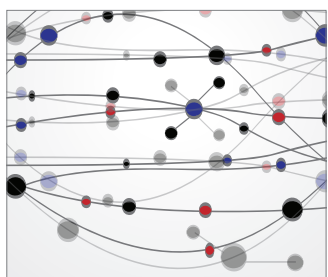

The Scientific World Journal
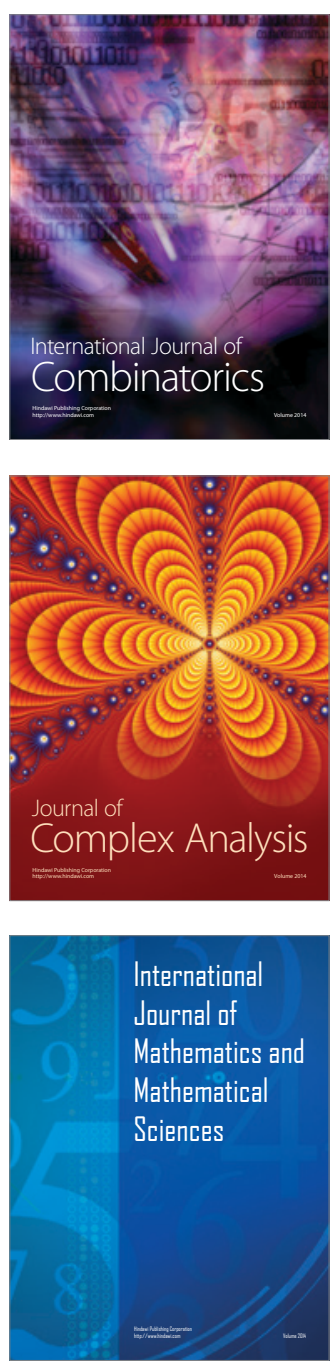
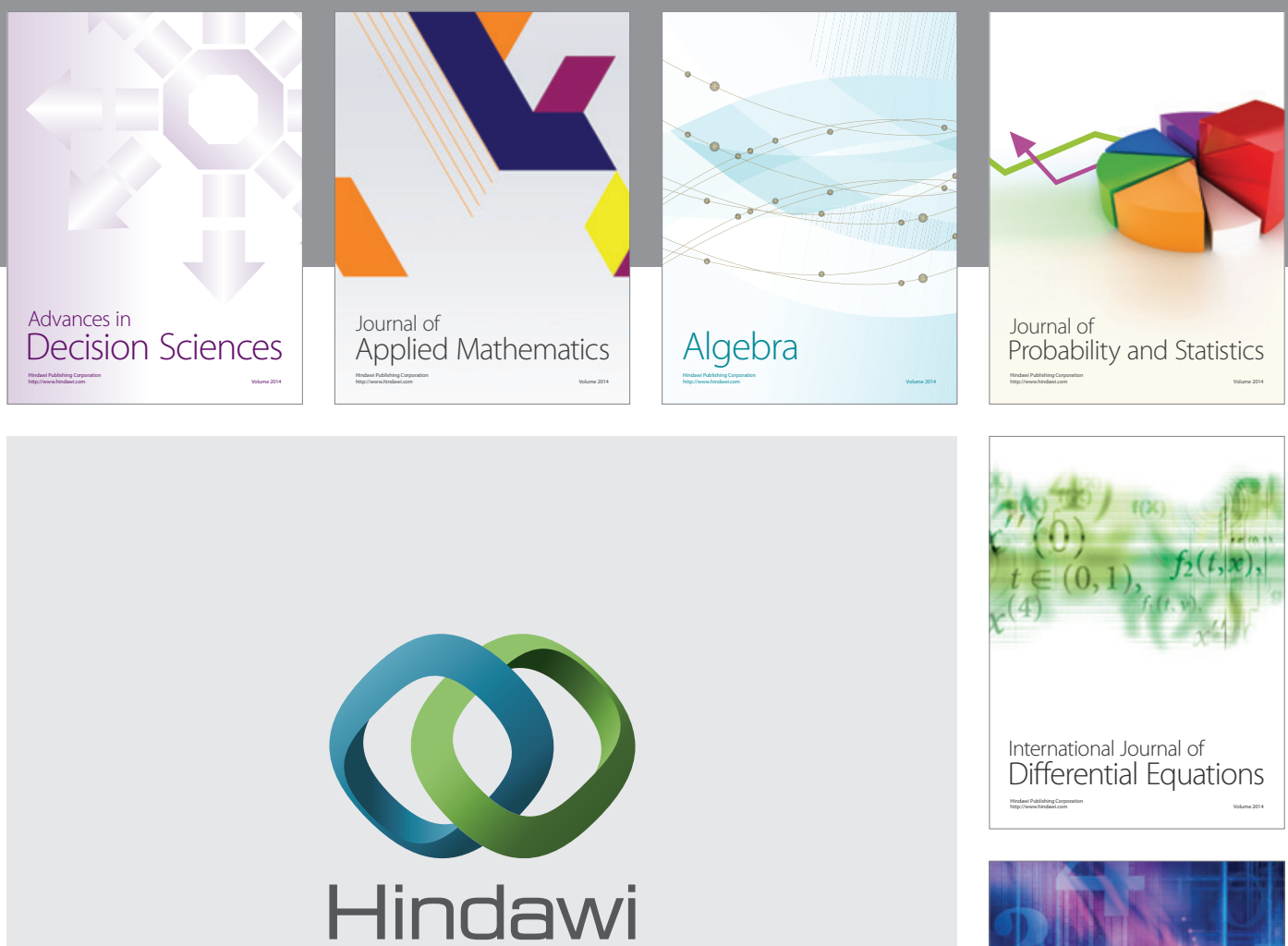

Submit your manuscripts at http://www.hindawi.com
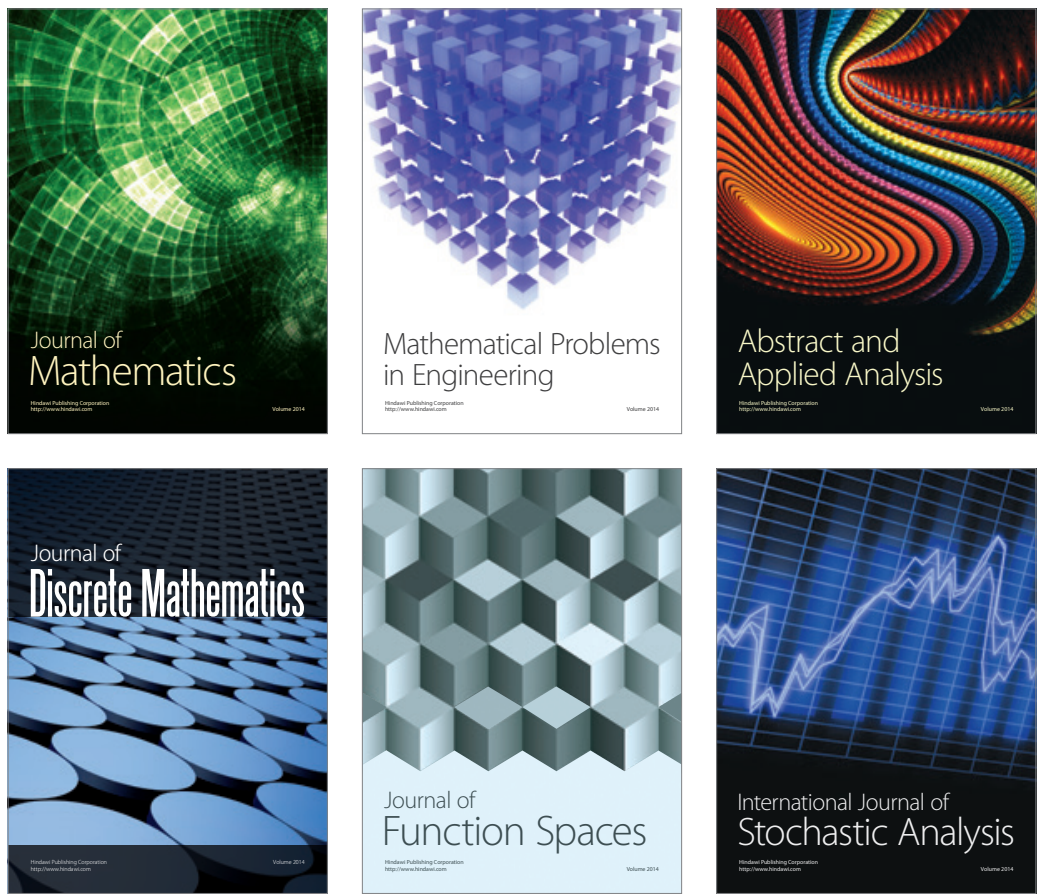

Journal of

Function Spaces

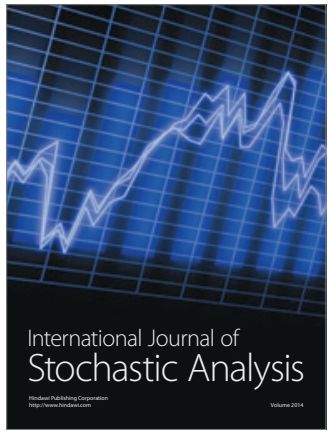

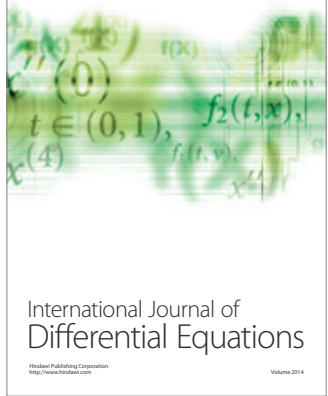
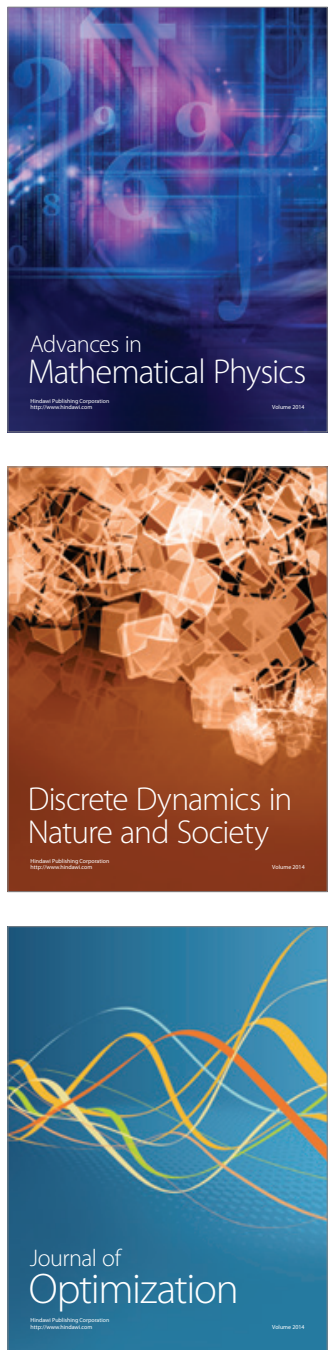